\title{
CT Features of Extrahepatic Growing Hepatocellular Carcinoma Located in the Portacaval Space
}

\author{
Yanming Ge, Yingshan Tu, Qianqian Wang, Xizhen Wang, Peng Dong* \\ Medical Imaging Center of the Affiliated Hospital, Wei fang Medical University, Weifang, China \\ geyanming2001@163.com \\ *corresponding author: Dong Peng
}

Keywords: portacaval space; Extrahepatic growing; Liver; Tomography; X-ray computed.

\begin{abstract}
To evaluate the computed tomography (CT) features of the extrahepatic growing hepatocellular carcinoma (HCC) located in the portacaval space. Eleven cases of extrahepatic growing hepatocellular carcinoma in the portacaval space were reviewed retrospectively which were proved by clinical and laboratory findings. Emphasis was placed on the CT features of the masses. 11 cases invaded all the portion of the portacaval space. 11 cases extrahepatic growing hepatocellular carcinoma arose from the liver and had no peduncle. The tumors showed enhancement in the early stage and quick return to hypodensity in the portal vein phase accompany with liver cirrhosis, splenomegaly and enlargement of the lymph nodes. CT is helpful in making accurate diagnosis of the extrahepatic growing hepatocellular carcinoma located in the portacaval space.
\end{abstract}

\section{Introduction}

In 1985, the literature [1] described the anatomical range between the portal vein and inferior vena cava as the porta-caval space (PCS). The portacaval space is anatomic deep and hidden. It is often difficult for conventional laparotomy to observe the situation of it.

This issue analyze the contrast-enhanced computed tomography (CT) findings of 11 cases of extrahepatic growing hepatocellular carcinoma located in the portacaval space retrospectively and discuss their characteristics on the CT images.

\section{Materials and Methods}

\subsection{Materials}

Subjects Retrospectively analyze eleven patients of extrahepatic growing hepatocellular carcinoma proved by clinical and laboratory findings located in the portacaval space. Eleven patients of extrahepatic growing hepatocellular carcinoma aged 35-56 years old (mean 45.2 years).

There were nine males and two females (Table 1).

\subsection{CT Examination CT scanning technologies}

Sixteen-dectector CT scanner (Siemens Sensation, Germany) was performed in all the patients, with the following parameters: $120 \mathrm{KV}-140 \mathrm{KV}, 210 \mathrm{mAs}-258 \mathrm{mAs}$ and 5mm slice width and 5mm reconstruction layer thickness. Scanning range includes the upper and middle abdomen. The Ultravist was injected intravenously via a high pressure syringe (300 mg I / mL, Bayer Schering Pharma AG, Berlin, Germany) at a rate of $2.5 \mathrm{ml} / \mathrm{s}$ to $3.0 \mathrm{ml} / \mathrm{s}$. The scan sequence included the hepatic arterial phase and the portal phase (CT scan was performed at 25 seconds to 30 seconds and 60 seconds to 65 seconds after the start of the injection). All the patients underwent CT enhancement scan.

\subsection{Observing Methods}

All the CT images were reviewed by two experienced radiologists with consensus who specialize 
in abdominal diseases.

When the radilolgists reviewed the CT images, the focus were on the site, shape, features of enhancement of lesions in the portacaval space and its complications.

\section{Results}

11 cases in the group of hepatocellular carcinoma arose from the caudate lobe, sessile, the size of $61 \mathrm{~mm} \times 50 \mathrm{~mm}$ to $145 \mathrm{~mm} \times 91 \mathrm{~mm}$; which enhanced in the early stage and quick return to hypodensity relative to the neighboring liver tissue. There were other intrahepatic tumors.

Cirrhosis and enlarged spleen were shown in the 11 cases, which also accompanied by increased retroperitoneal lymph nodes (Figure 1 and Figure 2).
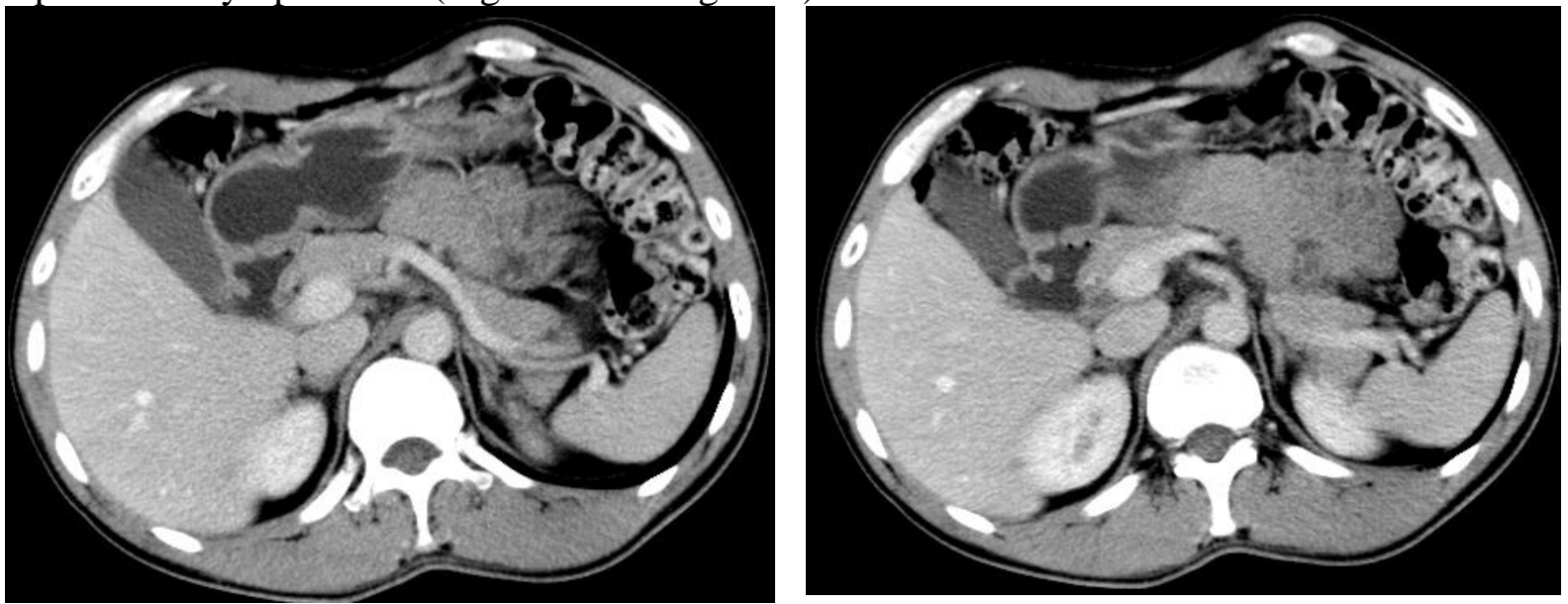

Figure1. Female, 48 years old. The axial CT images showed the normal portacaval space.

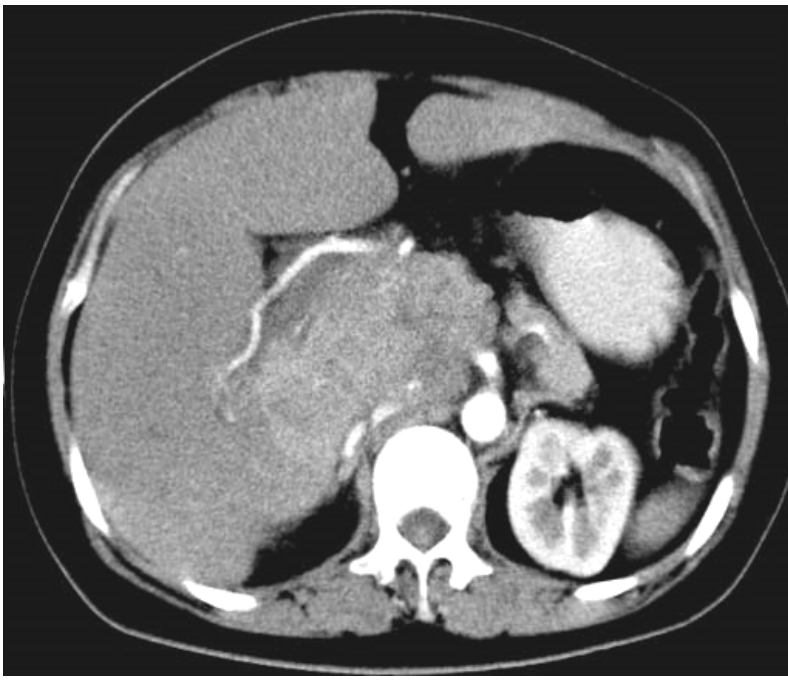

$\mathrm{a}$

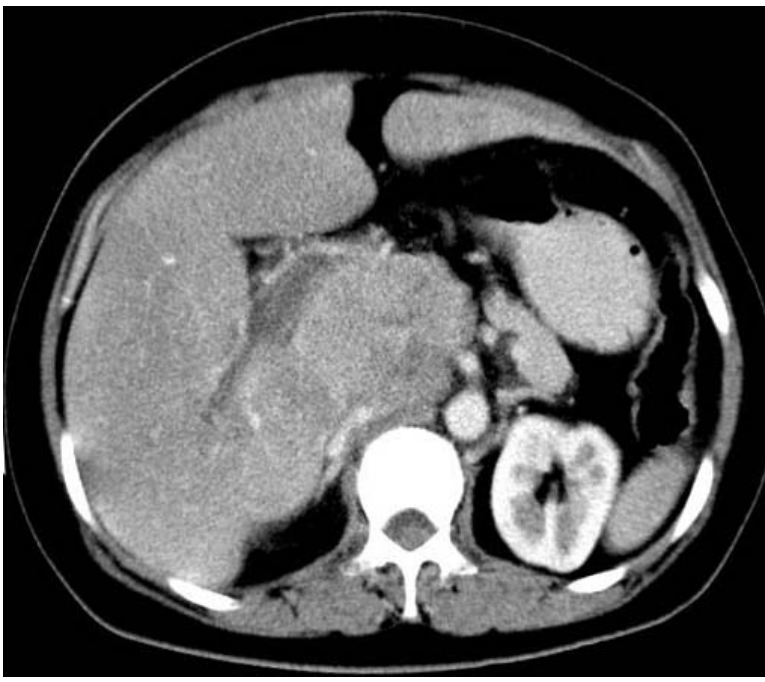

b

Figure 2. Female, 47 years old, extrahepatic growing hepatocellular carcinoma located in the portacaval space. Figure 2a and Figure 2b The CT iamges showed the mass located in the portacaval space arose from the hepatic caudate lobe (arrow). The tumor enhanced in the early stage and quick(Figure 2a) return to hypodensity in the portal vein phase(Figure2b).

\section{Discussion}

Extrahepatic growing hepatocellular carcinoma is the tumor that grow out of liver, only connect the liver with peduncle or close to liver. Mass can be spherical or eggplant-shaped protruding from the liver surface or be extrahepatic, which ranging in size. The majority of lesion and the liver are connected with peduncle, in a small number of cases, the tumor mass is close to the liver surface 
without sign of peduncle. Extrahepatic growing hepatocellular carcinoma may be associated with ruptured hemorrhage and retroperitoneal lymphadenopathy or ascites [2-4].

Most cases show typical CT manifestations of cirrhosis, even with multiple low-density nodules and retroperitoneal lymph nodes or a small amount of ascites. Extrahepatic growing hepatocellular carcinoma is divided into simple and mixed types according to whether the liver has tumors [4].

In this study, 11 cases of hepatocellular carcinoma arose from the caudate lobe, and the mass closed to the liver surface. Hepatocellular carcinoma showed enhancement in the early stage and quick return to hypodensity in the portal vein phase on the CT images. All the 11 cases had cirrhosis and spleen enlargement, accompanied with enlarged retroperitoneal lymph nodes.

Portacaval space can have a variety of blood vessels, mainly for the abnormal right hepatic artery and the branch of pancreatic duodenal vascular arch. Sometimes, the cystic duct can be shown in the portal space, omental hole is the potential cavity in the portacaval space. A part of the uncinate process of the pancreas may locate within the portacaval space. The superior part of the portacaval space is mainly composed of hepatic caudate lobe, and the inferior parts mainly composed of lymph nodes, portacaval blood vessels and the cystic duct which go through the portacaval space. All these are helpful for radiologists to identify the portacaval space and the processus caudatus and processus papillaris of caudate lobe on the CT and MRI images[1, 5-7].

The major tumor of hepatocellular carcinoma can be located in the center of the portacaval cavity, left or right part, and it is often located in the superior part of portacaval space. Hepatocellular carcinoma invade the portacaval space through many ways: primary carcinoma arises from right superior part of liver can directly invade the caudate lobe so that involve the portacaval space, primary carcinoma arises from right inferrior part of liver can invade the portal space by surrounding the inferior vena cava.

When the caudate lobe become the primary tumor of hepatocellular carcinoma, the lesion within the portacaval space is a part of the primary tumor. In these cases, the lesion within the portacaval space directly connect with the primary tumor. Hepatocellular carcinoma adjacent to the portacaval space often involve hepatoduodenal ligament and the portacaval space via the sapce between the vena or firstly invade hepatogastric ligament, and then involve the portacaval space through the hepatoduodenal ligament. so it shows that the lesions within the portacaval space connect the primary tumour by the hepatoduodenal ligament $[8,9]$.

In this group, 11 cases of extrahepatic growing hepatocellular carcinoma were involved the whole portacaval space, which may help to antidiastole the tumor which mainly grow in the portacaval space.

A variety of abdominal tumors may involve the portacaval space $[7,10]$. The major part of the duodenal cancer is located in the center or the right side of the portacaval space, especially the superior part. The way of enhancement is different from hepatocellular carcinoma. Cancer happens in the head of pancreas locate in the center or the in the inferior part of portacaval space. According to the anatomical basis that the portal vein is in the head of the pancreas, the pancreatic head cancer may directly violate the portacaval space from top to bottom.

The enhancement characteristic of Pancreatic head cancer is different hepatocellular carcinoma[6]. Extrahepatic growing hemangioma refers to the mass mainly grow outward the liver, and it prefers to occurring in the thinner places rather other parts [11]. The contrast-enhanced CT findings of the hepatic hemangioma is different from the hepatocellular carcinoma.

In one word, the contrast-enhanced CT examination is helpful to evaluate the extrahepatic growing hepatocellular carcinoma (HCC) located in the portacaval space.

\section{Acknowledgements}

This work was supported by a grant from the Shan-dong provincial natural science foundation of china(ZR2014HL083 and ZR2010HM078), Research topic of innovation program in graduate education in Shan-dong Province (SDYY15119), Research program of higher medical education research center of Shan-dong Province (YJKT201514), Project of medical education branch of the Chinese Medical Association (2016B-YJS031). 
Table 1 Clinical findings of the 11 cases

\begin{tabular}{|c|c|c|c|}
\hline case & gender & age & Anatomic location \\
\hline 1 & male & 35 & portacaval space \\
\hline 2 & male & 39 & portacaval space \\
\hline 3 & male & 40 & portacaval space \\
\hline 4 & male & 42 & portacaval space \\
\hline 5 & male & 46 & portacaval space \\
\hline 6 & male & 47 & portacaval space \\
\hline 7 & male & 48 & portacaval space \\
\hline 8 & male & 47 & portacaval space \\
\hline 9 & male & 56 & portacaval space \\
\hline 10 & female & 50 & portacaval space \\
\hline 11 & female & 47 & portacaval space \\
\hline
\end{tabular}

\section{References}

[1] Zirinsky K, Auh YH , Rubenstein WA, Kneeland JB, Whalen JP, Kazam E. The Portacaval Space :CT wit h MR Correlation. Radiology 1985 , 156 (2):453-460.

[2] Yang ZD, Xiang QY, Wang DY. Extrahepatic growing cancer: A case report. Chinese Hepatology 2002, 7(2) :144-144.

[3] Zhang HB, Yang GS, Lu JH, et al. Huge pedunculated hepatocellular carninoma and its surgical treatment. Chin Hepatobiliary Surg 2004, 10 (9):589-591.

[4] Chen MX, Huang NX, Zhou RM. CT diagnosis of pedunculated hepatocellular carcinoma (a report of 7 cases). J Prac Radiol 1998, 14(5): 282-283.

[5] Xie N, Min PQ, Chen WX, et al. CT and MRI evaluation of portacaval space: normal manifestations and clinical implications. Radiol Practice 2006, 21(5): 459-463.

[6] Min PQ, Lin S, He ZY, Song B, Zhou XP, Yang ZG, et al. A study on CT and MRI features of portacaval space abnormalities. Chin J Radiol 1996, 30(1): 6-10.

[7] Wang QQ, Wang YQ, Yang CB, Dong P. MDCT manifestations of normal portacaval space and Anatomic Basis. Chin J Clin Anat 2016, 34(5):514-516.

[8] bdom inal lymph nodes identified by computed tomography. Gastrointest Radiol 1988, 13(3):247-250.

[9] Mori H, Aikawa H, Hirao K, Futagawa S, Fukuda T, Maeda H, Hayashi K. Exophytic spread of hepatobiliary disease via perigepatic ligaments: demonstration with CT and US. Radiology 1989, 172(1): 41-48.

[10] Zhu YC, Guo YT, Wang JL, Zhu M, Shen JF. Multi-slice CT finding of upper abdominal tumor involved in portacaval space. J Chin Pract Diagn Ther 2015, 29(11): 1131-1132.

[11] Liang W, Jia HS, Zeng S, et al. CT diagnosis of pedunculated hepatic cavernous hemangioma: 4 cases report. Journal of Diagnostic Imaging \& Interventional Radiology 2002, 11(2): 111-112. 